\title{
Estrategias de participación e interacción en entornos virtuales de aprendizaje
}

\author{
Claudia Vásquez Lopera ${ }^{* \star}$ \\ Sandra Arango Vásquez ${ }^{* * *}$
}

Recibido: 20 de febrero de 2012 Aprobado: 27 de marzo de 2012

\begin{abstract}
Resumen
Este artículo surge de la investigación "Interacciones comunicativas en un entorno virtual de aprendizaje", realizada por el grupo de investigación E-Virtual. En ella el propósito era responder cómo se están generando las interacciones comunicativas y la participación de los estudiantes en un entorno virtual. La investigación se desarrolló mediante la metodología compresiva con enfoque etnometodológico a partir de los diálogos y discusiones en los foros, chat y mensajes, herramientas desde los cuales se comprendió que algunas estrategias de participación e interacción utilizadas por los estudiantes se relacionan con el horario, el orden de envío de las actividades, la solicitud de asesoría, el uso de las netiquetas, la co-evaluación y los silencios en el entorno. Aunque los hallazgos están relacionados con los estudiantes participantes de la investigación, es posible que se conviertan en características comunes a otros grupos de estudiantes participantes en entornos virtuales de aprendizaje.

Palabras clave: Participación, interacción comunicativa, entorno virtual, tecnologías de información y comunicación
\end{abstract}

El artículo es producto del proyecto de investigación "Interacciones comunicativas en un entorno virtual de aprendizaje" del grupo E-Virtual adscrito a la línea de investigación "Entornos Virtuales; financiado por la Vicerrectoría de investigación de la Universidad de Medellín. Investigadora principal: Sandra Isabel Arango Vásquez; Co-investigadoras: Claudia Patricia Vásquez Lopera, Angélica Ricaurte Avendaño; Auxiliares: Nancy Yaneth Gómez Valencia, Lorena Correa Correa. Realizado entre 2009 y 2011.

.* Maestranda en Educación y Desarrollo Humano. Grupo de investigación E-Virtual, Universidad de Medellín, Colombia. cpvasquez@ udem.edu.co

*** Magíster en Educación. Líder del grupo de investigación E-Virtual, Universidad de Medellín, Colombia. sarango@udem.edu.co

Anagramas Volumen 10, №20 pp. 95-108 ISSN 1692-2522 Enero-Junio de 2012. 228 p. Medellín, Colombia 


\section{Introducción}

Una de las políticas del Ministerio de Educación Nacional es la incorporación de las Tecnologías de Información y Comunicación (TIC) en los procesos académicos; para atender a ello, las Instituciones de Educación Superior (IES) están utilizando los Entornos Virtuales de Aprendizaje (EVA). Un EVA permite publicar documentos, realizar asesorías, realizar actividades evaluativas, organizar los espacios académicos, matricular y establecer la interacción comunicativa entre los participantes.

La interacción comunicativa es un elemento esencial en los procesos educativos, tanto presenciales como mediados por un EVA. Una de las preocupaciones del grupo de investigación es cómo se están generando estas interacciones y la participación entre los estudiantes en el espacio virtual.

La sistematización de las experiencias investigativas de la Fundación Universitaria Católica del Norte en su trayectoria como única IES colombiana virtual (Sánchez, et. al., 2010) y el estado del arte de 145 documentos sobre educación virtual (Restrepo et al., 2009) son algunos de los referentes conceptuales que nos permitieron sustentar o refutar el análisis categorial y la interpretación de los hallazgos, específicamente en las estrategias de participación e interacción en un EVA. Para el chat utilizamos como base el análisis de la oralidad, la escritura, la textualidad, los microelementos de sociabilidad online, descritos por Mayans (2002).

Algunas de las investigaciones sobre interacciones comunicativas están estrechamente relacionadas con comportamientos sociales, relaciones interpersonales en el aula de clase e interacciones comunicativas en el ámbito educativo; sin embargo, estas investigaciones no contemplan cómo se manifiestan las estrategias de participación en un EVA. El estudio de estas estrategias permite plantear recomendaciones para mejorar las prácticas comunicativas y los procesos académicos en la educación mediada por EVA.

La investigación "Interacciones comunicativas en un EVA" la realizamos mediante metodología comprensiva con enfoque etnometodológico. Los instrumentos utilizados fueron la observación directa, la revisión documental y el grupo focal. La ruta de análisis que utilizamos fue el registro de diálogos y conversaciones, codificación, análisis de referentes, recodificación y categorización.

Entre los diálogos y conversaciones registradas en el chat y los foros, analizamos: momentos de interacción, solución de inquietudes académicas, elementos de cortesía y procesos de co-evaluación utilizados por los participantes. Además, dos de los axiomas planteados por Watzlawick et al.,(1971), "No es posible no comunicar" y "La naturaleza de una relación".

Consideramos que un participante dentro de una interacción comunicativa es aquel que emite o percibe un mensaje; por lo tanto, en el EVA podemos evidenciar la noción de participar como el acto de un sujeto (estudiante) por ser visible frente a los otros, por mostrar frente al grupo de compañeros su aprendizaje, su conocimiento o su posición frente a un tema o discusión.

Dentro de las estrategias de participación e interacción analizamos los espacios de comunicación: chat y foros.

El chat es un espacio de comunicación sincrónica que permite la participación intergrupal entre los participantes y se caracteriza por la simultaneidad de los mensajes en el tiempo. Para la conversación en el chat, cada estudiante asumió un rol (líder, crítico-evaluador, relator). La participación a través de este espacio nos permitió observar, analizar, interpretar y finalmente comprender una gran cantidad de aspectos que se tejen alrededor 
de la participación académica, mediados por un espacio de comunicación sincrónica.

El foro es un espacio de comunicación asincrónica que permite la participación interpersonal e intergrupal; en el EVA los estudiantes tenían varios foros para resolver inquietudes y/o realizar actividades académicas durante el semestre.

En los foros, cuando la relación era intergrupal, quienes debían enviar las actividades evaluativas eran los relatores del equipo de trabajo. Sin embargo, todos los estudiantes tenían acceso al foro para visualizar el trabajo de los demás y enviar sus comentarios.

A partir del análisis de los diálogos y conversaciones, comprendimos que algunas de las estrategias de participación e interacción utilizadas por los estudiantes se relacionan con el horario nocturno, el orden en el envío de actividades, la solicitud de asesorías presenciales, el uso de netiqueta según la herramienta de comunicación, la co-evaluación como una estrategia de participación propiciada por la profesora y los silencios en el EVA como una forma de comunicar.

En este artículo presentaremos la metodología y los principales resultados, lo cual permitirá identificar estrategias de participación e interacción en un EVA, para diseñar un plan de mejoramiento de la comunicación mediada por este entorno virtual de aprendizaje.

\section{Metodología}

Esta investigación es cualitativa o comprensiva, y parte del enfoque etnometodológico definido por Garfinkel; su mayor aporte está en el estudio del lenguaje natural de las relaciones interpersonales que se entretejen en los diálogos y conversaciones que comparten los sujetos, en este caso, en las relaciones académicas de los estudiantes.
Los etnometodólogos atribuyen mucha importancia al lenguaje natural, visto como un sistema de prácticas que les permite a las personas participar en la producción y la realización de la vida social y al mismo tiempo presenciarlas. El lenguaje natural es el conjunto de elementos no lingüísticos de la comunicación interpersonal. Incluye, por ejemplo, establecer y respetar los turnos en la conversación, superar las interrupciones, centralizar el uso de la palabra. Es decir, su estudio conlleva a analizar la estructura básica de la interacción entre el emisor y el receptor (Galeano, 2004:153).

La relación de los participantes de la asignatura TIC mediada por el EVA era propiamente académica y se realizó a través del lenguaje escrito. A diferencia de las grabaciones de audio y vídeo, aquí la interpretación de la kinésica, el tono de voz y otros rasgos propios de la relación cara a cara pueden estar representados por signos, símbolos y líneas de texto.

Para el estudio del lenguaje, fue necesario el aporte de la pragmática para interpretar la intencionalidad del emisor y el efecto que podía causar en el perceptor; la semiótica para la interpretación de los signos dentro del texto y el interaccionismo simbólico para reconocer la interiorización de los símbolos que se están construyendo en las interacciones comunicativas dentro de un EVA; y los aportes de Austin cuando hace referencia a los actos locutivo, ilocutivo y perlocutivo.

La unidad de análisis la conformaron 16 estudiantes de la asignatura electiva TIC (metodología blended) de la Universidad de Medellín, en el semestre 2009-1. Los 16 estudiantes conformaron 7 equipos de trabajo ( 5 equipos de 2 integrantes, y 2 equipos de 3 integrantes) para realizar un proyecto de aula de investigación formativa.

Los instrumentos utilizados en esta investigación fueron la observación directa, la revisión documental y el grupo focal. La observación direc- 
ta la realizamos como participantes matriculados dentro de la plataforma, actuando como sujetos observantes con acceso a los diálogos y conversaciones; utilizamos fichas de observación para registrar las evidencias e ir codificando los datos. La revisión documental la realizamos en un formato que permitió registrar investigaciones, textos, experiencias y comentarios de los investigadores. El grupo focal (14 estudiantes), nos permitió conocer las percepciones de los estudiantes frente a la experiencia y validar las interpretaciones registradas en las fichas de observación.

La ruta de análisis comenzó con la codificación de los diálogos y conversaciones; después se clasificaron los códigos iniciales y emergentes, permitiendo una depuración de los datos (re-codificación). Asimismo, se contrastaron los referentes conceptuales del formato de revisión documental con los códigos de análisis. Finalmente, se interpretó la información con los mapas de categorías.

\section{Discusión y resultados}

\section{Axioma: "No es posible no comunicar"1}

... si se acepta que toda conducta en una situación de interacción tiene un valor de mensaje, es decir, es comunicación, se deduce que por mucho que uno lo intente, no puede dejar de comunicar. Actividad o inactividad, palabras o silencio, tienen siempre valor de mensaje: influyen sobre los demás, quienes, a su vez, no pueden dejar de responder a tales comunicaciones y, por ende, también comunican (Watzlawick, et al., 1971:50 citado por Vásquez y Arango, 2010:133).

Desde la mirada de Watzlawick, et al.,(1971) en el axioma "no es posible no comunicar"; ana-

1 El análisis que presentamos en este ítem incluye apartes de los resultados parciales de la investigación publicados en: Vásquez Lopera, Claudia Patricia; Arango Vásquez, Sandra Isabel (2010:133-137). Capítulo 7: Interacciones comunicativas en un Entorno Virtual de Aprendizaje (EVA). En: Pensar la comunicación - Reflexiones y resultados de investigación, Tomo II. Medellín: Sello Editorial Universidad de Medellín. lizamos el silencio en la comunicación por fallas en la conexión, el uso de zumbidos en el chat, el uso simultáneo de herramientas digitales, la preparación previa del chat y la manipulación del registro de textos del chat.

La ausencia en el envío de mensajes, o lo que consideramos como un "silencio" en la comunicación, es un aspecto de análisis que puede reflejar un inconveniente técnico o académico.

Algunas de las secuencias comunicativas entre los participantes se vieron interrumpidas por fallas en la conexión, lo que causó el efecto de finalización abrupta de la comunicación o una pausa de la interacción que más adelante fue retomaba en forma planeada o fortuita.

La comunicación a través de la herramienta sincrónica chat permite el uso de zumbidos; en el caso de la asignatura electiva TIC, los participantes interpretaron este sonido como una forma de comunicarse con el otro indicando una demora en la respuesta o una falta de coherencia en el acto comunicativo. El zumbido realizado por un emisor puede tener una intencionalidad diferente a lo que percibe el otro y en ningún momento lo interpretamos fuera del contexto.

En el manejo simultáneo de algunas herramientas digitales, los estudiantes manifestaron que enviaron mensajes por correo electrónico, transfirieron archivos, buscaron información en Internet, entre otros; todo esto mientras estaban en la sesión de chat con su equipo de trabajo. Tal como lo indica Mayans en su libro Género chat: "El ritmo y la estructura de participación son variados e irregulares, puesto que cada interactuante puede estar sometido a otros estímulos online y offline, y estos pueden ir variando" (Mayans, 2002:114).

En la preparación previa del chat, encontramos que algunos de los participantes tuvieron una sesión de pre-chat, es decir, ya habían conversado y planeado unas estrategias para la conversación; 
esto nos permitió observar que el segundo chat lo realizaron más organizado. Consideramos que esta estrategia puede mejorar la coherencia, el orden y la calidad de la conversación sincrónica. Un equipo de trabajo optó por realizar varios encuentros de chat, permitiendo que cada integrante pudiera mejorar sus aportes antes de compartirlos con los demás compañeros.

En la manipulación del registro de textos del chat, encontramos que los participantes de un equipo de trabajo borraron las líneas de conversaciones personales o informales que no correspondían a la actividad académica.

\section{Axioma: "La naturaleza de una relación"}

Este axioma "se refiere a la interacción -intercambio de mensajes- entre comunicantes. Para un observador, una serie de comunicaciones puede entenderse como una secuencia ininterrumpida de intercambios. Sin embargo, quienes participan en la interacción siempre introducen lo que, siguiendo a Whorf (1956), ha sido llamado por Bateson y Jackson la "puntuación de la secuencia de hechos (Watzlawick, et al., 1971:50 citado por Vásquez y Arango, 2010:139).

Este axioma lo aplicamos en el análisis del foro, en cuanto al uso que le dieron los estudiantes para el envío de actividades, leer y enviar comentarios.

En algunas actividades académicas los estudiantes debían enviar un trabajo al foro y realizar comentarios al trabajo de los compañeros. Por ser el foro una herramienta asincrónica donde la información permanece pública, los estudiantes tenían un rango de tiempo para participar e ir observando lo que hacían los demás, aquí encontramos que:

- Son comunes los estudiantes que envían el trabajo primero, pensando en que la calificación será menos exigente; otros, por el contrario, esperan que la mayoría lo envíen para así poder mirarlo y aplicar algunos elementos en su trabajo. En otro caso intermedio, algunos equipos preferían revisar los parámetros del trabajo indicados por la profesora antes de enviarlo y no aplicaban ninguna estrategia en el orden de la participación. En esta experiencia fue notable que cuando el primer estudiante o equipo de trabajo enviaba la actividad incorrecta, los demás se basaran en ello y en su mayoría también cometieron errores similares.

- En algunos foros evidenciamos poca participación de los estudiantes; la mayoría de los estudiantes que tenían el rol de "relator" adoptado en la primera actividad del chat fueron quienes se responsabilizaron de enviar los trabajos en la plataforma. En cuanto al orden de la secuencia de actos comunicativos, en algunos casos estuvo limitada al relator y a la respuesta de la profesora con la valoración de la actividad.

- Hubo poca interacción comunicativa en los foros; la mayoría de los estudiantes enviaban comentarios como parte de la actividad calificable. De los estudiantes que se limitaron a enviar los trabajos académicos, podemos decir que su aprendizaje estuvo más centrado en el contenido que en otros elementos inherentes al proceso, como son la participación, argumentación y lectura del trabajo de los compañeros.

- El foro inquietudes fue utilizado por algunos estudiantes que estaban más inmersos en la dinámica de participación, así, en este espacio podían encontrar posibles soluciones a sus dudas a través de preguntas enviadas públicamente por otros compañeros.

- La participación de un estudiante en el foro, donde indicaba fallas y recomendaciones al trabajo de un compañero, propició la participación de otros estudiantes en el mismo foro. Esta es una aplicación de la co-evaluación en entornos virtuales de aprendizaje, la cual puede o no ser parte de la actividad calificable. 


\section{Momentos de interacción en el EVA}

Una de las características de los EVA es la posibilidad de acceso durante los 7 días de la semana, las 24 horas del día (24/7). Los estudiantes que tienen dificultades con horarios fijos de estudio y desplazamiento son los que generalmente seleccionan la educación bajo metodología virtual.

Sin embargo, en la asignatura electiva TIC los estudiantes están matriculados bajo la modalidad presencial pero están aplicando la metodología blended. De ellos también conocimos las preferencias de horario para publicar las actividades evaluativas en la plataforma, para enviar comentarios en los foros, para comunicarse con los compañeros a través de la herramienta "mensajes", y para realizar la actividad del chat.

Cuando indagamos en el grupo focal sobre las ventajas y desventajas que los estudiantes habían encontrado en la utilización del EVA en la asignatura electiva TIC, ellos afirmaron que una ventaja es la flexibilidad en el tiempo para entregar las actividades académicas, de manera que no interfiere con actividades de otras asignaturas.

Un estudiante considera que la flexibilidad de los horarios en un EVA puede convertirse en un riesgo de no acordarse de la fecha límite de entrega de las actividades calificables.

Adicionalmente, analizamos la percepción de los estudiantes en la efectividad de los tiempos de respuesta. Tomemos como referente el siguiente caso:

Cuando un estudiante afirma que "la profesora siempre nos respondía a tiempo" o que los compañeros no reciben los mensajes en un "tiempo oportuno", no es que haya un límite de horas para responder un mensaje de manera que podemos garantizar la efectividad de la comunicación, sino que el factor tiempo en este caso es relativo a la necesidad del estudiante. Por ejemplo:
Una actividad académica tiene como fecha límite de entrega el jueves a las 23:55, el estudiante comienza a hacer la actividad ese mismo día a las 21:00. Realizando el trabajo, el estudiante tiene una inquietud, aprovechando la flexibilidad de tiempo 24/7 de la plataforma e-learning, envía un mensaje al profesor a las 22:50 (puede ser un mensaje privado o a través del foro inquietudes), ¿será que el profesor está disponible a esa hora para responder su inquietud y poder así terminar el trabajo?

En este ejemplo, el estudiante requiere una respuesta inmediata para finalizar su trabajo y enviarlo en el tiempo límite definido. Por lo tanto, para este estudiante, la efectividad en el tiempo de respuesta es de aproximadamente 1 hora.

Por el contrario, si tenemos un caso donde el estudiante comienza a resolver la misma actividad académica desde el jueves anterior (8 días antes) y envía la inquietud a la plataforma el lunes, estamos hablando de un "tiempo oportuno" de respuesta por parte del profesor de 3 días.

Específicamente, los momentos de realización del chat, de la participación en los foros y del envío de mensajes fueron:

\section{a) Momentos de realización del chat académico}

La actividad académica que realizaron los equipos de trabajo a través del chat debía ser enviada a la plataforma el día 15 de marzo a las 23:55, según los momentos registrados en la plataforma e-learning; la mayoría de los equipos realizaron esta actividad a partir de las 1 1:00 del último día de plazo de entrega y comenzaron a enviarlo a la plataforma e-learning después de las 18:00. Solo un equipo evidenció varios encuentros en el chat en fechas anteriores al momento de entrega.

\section{b) Momentos de participación en los foros}

En los registros de participación, encontramos que los estudiantes tienen preferencia para ingresar en horas nocturnas. El caso más extremo 
fue el de un participante que envió comentarios al foro a la 1:26, los demás estuvieron en el rango 20:24 a 00:38.

Es de anotar que con esto no queremos indicar generalización de los datos, pero no hay que desconocer que se puede convertir en una característica común entre los estudiantes para participar en un EVA.

\section{c) Momentos de interacción comunicativa a través de la herramienta mensajes}

Aunque la herramienta mensajes es asincrónica, la interacción comunicativa de los estudiantes a través de la herramienta mensajes se presentó principalmente en encuentros sincrónicos; es decir, cuando un compañero ingresaba al EVA y veía a un compañero conectado, lo consideraba una posibilidad de inicio de conversación.

\section{Sobre la solicitud de asesoría e inquietudes}

En cuanto a la solicitud de asesorías e inquietudes, analizamos la forma como se presentaron en el EVA. Encontramos que los estudiantes hacían sus preguntas de manera pública a través de los foros, o de manera privada a través de mensajes. En los foros, la profesora o los compañeros de la asignatura podían intervenir en la relación comunicativa y enviar la respuesta a un estudiante.

Aunque una característica de la metodología virtual es la mediatización del proceso de formación del estudiante mediante el EVA, encontramos que hay una preferencia de los estudiantes para asistir a las asesorías presenciales.

Cuando las inquietudes se planteaban públicamente en el foro, la respuesta no era considerada una función o responsabilidad de la profesora, por el contrario, académicamente se considera una construcción colectiva que apunta al aprendizaje de todos.
Aunque en la interacción en el aula el profesor juega un papel importante (como mediador en el aprendizaje) no es menos importante el papel de mediación que desempeñan los compañeros de aula, tanto en los procesos de socialización como en el desarrollo cognitivo. En efecto, las experiencias de aprendizaje cooperativo repercuten positivamente en el establecimiento de relaciones abiertas y profundas entre los alumnos, y en el rendimiento y productividad de los participantes. El aprendizaje compartido, además favorece la metacognición (Medina y Salvador, 2002:179).

En el chat académico también se presentaron inquietudes sobre el desarrollo de las actividades; en este caso fueron todas dirigidas en forma sincrónica al (los) compañero (s) del equipo de trabajo. Los estudiantes utilizaron también el espacio de mensajes de la plataforma para resolver inquietudes académicas.

No necesariamente cada vez que un estudiante tenía una inquietud podía obtener la respuesta. Para Austin existen circunstancias en el acto comunicativo que se consideran expresiones realizativas infortunadas, tal como se pudo conocer a través de algunas conversaciones.

\section{Elementos de cortesía en las interacciones comunicativas de los estudiantes en un EVA}

Se considera la cortesía en una interacción comunicativa como aquella representación del lenguaje que demuestra respeto y atención en la relación con el otro; puede presentarse al principio, durante el desarrollo y/o al final de una conversación.

Los elementos de cortesía que aquí analizamos no garantizan que la conversación sea fluida, ni que corresponda a un excelente desempeño en la actividad evaluativa, pero sí puede propiciar mayor interacción entre los participantes y generar efectos diferentes según las circunstancias en que se presenten. 
Austin considera que las acciones que se producen en una interacción comunicativa deben cumplir con unas reglas mínimas, lo que garantizará que exista una mayor probabilidad de respuesta o de causar el efecto esperado en el otro, lo que denomina "acto realizativo afortunado". La regla dice: "Tiene que haber un procedimiento convencional aceptado, que posea cierto efecto convencional; dicho procedimiento debe incluir la emisión de ciertas palabras por parte de ciertas personas en ciertas circunstancias" (Austin, 1962:56)

A continuación mostramos cómo se manifiesta la cortesía en el chat, el foro y los mensajes:

\section{¿Cómo es la manifestación de la cortesía en el chat?}

El chat, como herramienta sincrónica, cumple con requisitos mínimos de cortesía como son el saludo, la respuesta al saludo, la despedida, la respuesta a la despedida, el agradecimiento por una información o favor realizado por el otro, y la indicación explícita de que la conversación puede finalizar momentáneamente pero sigue abierta para otros momentos.

El saludo entre sujetos de un chat puede ser de varias formas; en las conversaciones de los estudiantes encontramos:

1) El saludo simple, correspondiente a un saludo corto, donde no se nombra al otro y se asume que es para todos los participantes del chat; por ejemplo: "Hola", "hellooooo", "hola niñas".

2) El saludo personalizado que va acompañado del nombre del otro.

3) El saludo incluyente, donde se saluda y se pretende conocer cómo se encuentra el otro sujeto puede hacerse en forma general o personalizada; la forma como se estructura este saludo queda a la espera de una respuesta; por ejemplo: "Hola, ¿cómo estás?", "¿Cómo les ha ido?", "¿Qué más xxxxx?", "Hola xxxxx ¿cómo estás?".

Sin importar el tipo de saludo que utilicen los participantes, consideramos que por la relación mediática que se presenta en el chat, el saludo constituye un aspecto relevante para iniciar una conversación; es una forma de verificar que técnicamente no existen problemas en la comunicación y hacen al sujeto un miembro visible dentro del equipo de trabajo.

El saludo cumple una función de visibilidad vehemente, de hacer patente la presencia del personaje. Tanto si el saludo es abierto les el que nosotros denominados simple] como si se dirige a alguien en concreto les el que nosotros denominados personalizado], su intención es la de hacerse visible y manifestar su voluntad de interaccionar con la concurrencia [aquí para nosotros es interaccionar con el equipo de trabajo] (Mayans, 2002, 106-107).

Igualmente, el análisis de las respuestas a los saludos permitió llegar a la siguiente clasificación:

1) Respuesta simple, puede darse inmediatamente después de un saludo o después de haber intercambiado varias líneas de saludo indicando la finalización de esta primera forma social de la conversación.

2) Respuesta personalizada, hace referencia directa al sujeto que inició el saludo.

3) Respuesta incluyente, se da cuando un primer sujeto ya saludó y el interlocutor considera relevante conocer cómo se encuentra el otro; esto indica que el saludo como forma social no ha finalizado y está a la espera de otra respuesta.

4) Respuesta formal, está más relacionada cuando el otro muestra agradecimiento frente al interés del compañero por conocer su estado. 
La respuesta al saludo indica que hay una correcta conexión en el chat y una disposición de comenzar la conversación sobre la temática convocada.

Con respecto a las expresiones de despedida de los sujetos en una conversación, también se pudo ver reflejada la cortesía de los participantes en el chat. La despedida se presentó cuando los estudiantes ya habían definido el último punto del trabajo académico; en forma general se pueden nombrar las siguientes características de despedidas:

1) Despedida simple: un ejemplo de este tipo de despedidas son las que utilizan palabras como "chao", "adiós", entre otras. Una característica es que no dejan abierta la posibilidad de re-encontrarse en una próxima conversación de chat; es lo que podríamos denominar la finalización de la interacción.

2) Despedida incluyente: este tipo de despedidas hace referencia a las expresiones donde el emisor refleja un "aparente" interés por el bienestar del "otro"; en este caso, se encontraron frases como "suerte", "que estés bien", entre otras.

3) Despedida abierta: este tipo de despedidas se caracterizan por una finalización temporal de la conversación, dejando abierta la posibilidad de un siguiente encuentro o una continuación de la interacción comunicativa entre los sujetos. Algunos ejemplos son las expresiones: "así quedamos, entonces", lo que presupone unos compromisos asumidos en la conversación que deben ser realizados al finalizar el encuentro del chat. Lo que no se puede afirmar es que la interacción comunicativa en este caso se retome nuevamente a través de la misma herramienta de comunicación (chat).

4) Despedida personalizada: corresponde a las despedidas donde se nombra directamente al otro, por ejemplo, "hasta luego, Lorena".
Sin embargo, en ninguno de los 7 equipos de trabajo analizados en esta investigación se presentó este caso.

En uno de los equipos de trabajo, no se presentaron las despedidas; es posible que estuviera incompleta la conversación del chat que los estudiantes pegaron en la agenda para presentarle a la profesora y que no presentaran las líneas de despedida, o el otro caso es que los participantes se desconectaron sin despedirse.

¿Cómo es la manifestación de la cortesía en los foros?

La cortesía en los foros la analizamos de forma similar al chat, teniendo en cuenta que esta herramienta es asincrónica y posiblemente refleje nuevas características.

Sánchez et al. (2010: 94) recomiendan aplicar los siguiente elementos dentro de la estructura esquemática o textual de un mensaje de un foro: 1) fórmula de saludo, 2) anuncio del contenido y contextualización de la situación, 3) el contenido del mensaje, 4) fórmula de despedida, 5) nombre del participante. Dentro de la valoración académica que hacen los docentes de esta estructura se encuentran los siguientes aspectos: - cortesía, - el grado de calidad y formalidad del lenguaje empleado (formalidad textual), - expresiones de actitud, - conocimiento / discurso especializado, - argumentación.

Para los autores, es necesario que los estudiantes conozcan este tipo de estructura y recomendaciones de cortesía como parte de su proceso de aprendizaje; no basta con decirles a los estudiantes "responda" o "participe"; es recomendable indicarles "cómo participar" o más bien, "cómo comportarse" en la interacción comunicativa a través de una herramienta específica.

"Resulta útil socializar con los estudiantes la estructura textual esperada en un foro educativo; 
es decir, los elementos que integran el mensaje y que evidenciarían un conocimiento pragmático textual de este género dialógico" (Sánchez et al., 2010:94)

Según los comentarios de los foros enviados por los estudiantes no fue una característica común entre ellos aplicar la estructura esquemática de cortesía; algunos casos analizados fueron:

- El saludo personalizado al responderle a un compañero en el foro indica que la respuesta está dirigida solamente a él, aunque tengan acceso todos los participantes.

- Un comentario sin saludo en el foro indica que no hay un perceptor específico.

- Un comentario sin saludo pero finalizando con la expresión "gracias" implica que el participante está agradecido por algo que indicó en el texto y asume que todos los demás lo leerán y aplicarán.

- Cuando un saludo en el foro inicia con expresiones como "buenos días", "buenas tardes" o "buenas noches" está asumiendo un encuentro sincrónico con el perceptor, lo que no es acorde con las características de la asincronía del foro.

- Un saludo simple en el foro refleja al destinatario sin necesidad de nombrarlo específicamente. Esto se visualiza dependiendo de la jerarquía de la herramienta.

¿Cómo es la manifestación de la cortesía en los mensajes?

La herramienta mensajes, aunque es asincrónica, se utilizó en muchos casos como sincrónica; el estudiante que comenzaba la interacción comunicativa lo hacía con un compañero que estuviera en línea en la plataforma e-learning en ese mismo momento.
Para indicarle a la otra persona la intencionalidad de comenzar una conversación, siempre comenzaban con un saludo (cualquiera de los que analizamos anteriormente, al fin y al cabo, saludo), pero al continuar con los mensajes las frases se convertían en parte de una conversación sincrónica omitiendo de antemano nuevamente el saludo.

Con respecto a las expresiones de agradecimiento en los mensajes, se manifestaron muy similares a las demás herramientas; simplemente se emitía la expresión "gracias" frente a una ayuda proporcionada por el compañero.

Para finalizar, consideramos que la cortesía, y muchos otros elementos de las interacciones comunicativas se manifiestan diferentes según el momento en que se realice, la intencionalidad y el rol de los sujetos participantes y, por ende, producen un efecto diferente en el perceptor.

Expresar las palabras es, sin duda, por lo común, un episodio principal, si no el episodio principal, en la realización del acto, cuya realización es también la finalidad que persigue la expresión [...] es menester que el que habla, o bien otras personas, deban también llevar a cabo otras acciones determinadas "físicas" o "mentales", o aun actos que consisten en expresar otras palabras (Austin, 1962:49).

\section{La co-evaluación como una estrategia de participación de los estudiantes}

La co-evaluación "es una forma de evaluación en la que pueden participar todos los individuos que conforman un equipo o grupo (docente, estudiantes); entre todos evalúan el comportamiento, participación y aprendizajes alcanzados. Para llevar a cabo actividades de co-evaluación se debe formar en la capacidad y disposición para evaluar a los compañeros y para ser evaluado por el mismo grupo, aspecto que se facilita en los entornos virtuales de aprendizaje (Restrepo, et al., 2009:46). 
La co-evaluación en la asignatura electiva TIC fue realizada a través de los parámetros de evaluación; cada estudiante debía visitar el foro para leer el trabajo de algunos compañeros y enviarles sus comentarios; después de eso la profesora enviaba la ficha de evaluación con las observaciones de cada ítem y con el resultado cuantitativo.

Con respecto a esta estrategia de participación en el foro, obtuvimos la siguiente clasificación:

1) Co-evaluación acertada: los comentarios enviados a los compañeros evidenciaban una lectura cuidadosa del trabajo y coincidían en gran parte con los comentarios realizados por la profesora.

2) Co-evaluación no acertada: este caso se presentó cuando un estudiante resalta el buen trabajo de un compañero y al ser revisado por la profesora encuentra que no cumple con las características indicadas para la actividad; esto demuestra una falta de lectura por parte del estudiante que envió un comentario no acertado.

\section{Conclusiones}

No toda la comunicación en el EVA está relacionada con el envío de mensajes textuales o icónicos; en algunos casos como en el chat, los estudiantes envían expresiones a través de "zumbidos". Concluimos que la intencionalidad con la que un estudiante emite un zumbido puede ser diferente a la que percibe el otro sujeto. El zumbido no es un ruido emitido por el chat sino que es algo que los sujetos comunicantes quieren expresar.

En el ámbito educativo pueden generarse "silencios" tanto desde la interacción comunicativa presencial como en la interacción comunicativa en EVA. Lo importante de esta situación es que el profesor reconozca el sentido del "silencio" en el desempeño de un estudiante.

Afirmamos que el saludo y su respuesta en el chat no evidencian la misma cortesía entre todos los participantes; esto es una característica perteneciente a un sujeto en particular.

La pregunta de un estudiante a través de una herramienta pública (de acceso para todos los participantes) como es el foro contribuye a disminuir la cantidad de inquietudes sobre un mismo tema. De esta manera, si un compañero tiene la misma inquietud, puede acceder a la respuesta sin necesidad de volver a preguntar lo mismo.

La despedida no fue un elemento de cortesía utilizado por los estudiantes en los mensajes, su uso de manera sincrónica puede indicar en los estudiantes que la interacción comunicativa finaliza simplemente cuando el otro compañero se desconecta de la plataforma e-learning; para ellos fue sin duda una decisión personal que no fue necesario comentarla con su compañero.

Cuando el foro es un espacio de comunicación utilizado para el envío de actividades académicas y en algunos casos para la co-evaluación, concluimos que la interacción comunicativa finaliza en el momento en que el profesor envía su valoración cuantitativa y/o cualitativa. Solo hay una continuación de la interacción comunicativa si los estudiantes expresan inconformidad sobre la valoración cualitativa y la nota asignada.

Ventajas del EVA en la metodología blended con respecto a la modalidad presencial:

- Todos los compañeros tienen acceso al trabajo de los demás y pueden elegir al final a cuál de todos le quieren realizar sus comentarios.

- La participación de cada estudiante queda registrada por lo menos hasta que finalice el periodo académico. 
- El profesor puede verificar el contenido y la calidad del comentario que realice cada estudiante.

- No se requiere que todos los participantes estén en un mismo lugar a la misma hora.

- El estudiante puede realizar comentarios más estructurados académicamente ya que el límite de tiempo no es de minutos sino de días.

- Un error en el comentario puede ser corregido, mejorado o borrado antes de que otro participante lo vea.

\section{Recomendaciones}

Una de las normas de Netiqueta para el uso del chat es indicar a los demás participantes la finalización de una idea; posiblemente eso ayude a mejorar la secuencia lógica de las ideas y a respetar el orden de participación. Esta norma puede ser propuesta grupalmente por el profesor y/o definida por los estudiantes dentro del equipo de trabajo antes de comenzar la sesión de chat. Ejemplos de esto son: utilizar una $\mathrm{T}$ al final para indicar que la intervención del participante ha finalizado "...o poner "..." (tres puntos sucesivos)..." para indicar que no se ha finalizado una idea.

Para las actividades evaluativas en el foro, recomendamos que el profesor incluya unos criterios específicos en la guía de evaluación, donde clarifique que los primeros trabajos publicados en la plataforma no son una pauta a seguir; cada estudiante o equipo de trabajo debe considerar únicamente los criterios consignados en la guía de evaluación.

Para un mejor aprovechamiento del foro, es recomendable que los profesores implementen estrategias didácticas, como por ejemplo, trabajo cooperativo y colaborativo; además, que promuevan la participación de los estudiantes en los foros como espacios de discusión no necesariamente en actividades calificable.

Proponemos que antes de realizar una sesión de chat académico, los estudiantes tengan claro su objetivo, ventajas y diferencias frente a lo que hacen generalmente en un chat social. Esto evitará conflictos en las relaciones por la falta de acuerdos.

El tiempo de mayor inquietud de los estudiantes es el que está más cerca de la entrega de una actividad académica; con base en esto se pueden programar las asesorías; por lo tanto, si el profesor no garantiza atención en la plataforma los fines de semana, le recomendamos programar la entrega de actividades en semana y asegurar una atención periódica a los estudiantes.

Consideramos que el nombre del participante al final del mensaje no es indispensable en herramientas como el foro, debido a que en el encabezado de la participación aparece claramente quién es el autor. Otra cosa muy diferente es si en vez de "nombre del participante" hablamos de la firma del autor, lo que podría implicar en algunos casos una posible diferenciación con el nombre registrado en la plataforma.

La co-evaluación es una práctica poco desarrollada por los estudiantes, que requiere de mayor motivación y seguimiento por parte de los profesores y que se debe propiciar con el fin de que los estudiantes reconozcan la importancia de sus aportes en el aprendizaje significativo de los compañeros. Recomendamos incluir la co-evaluación en los ítems de calificación para generar una cultura de participación crítica en el proceso de aprendizaje.

A manera de reflexión, es diferente la forma como se manifiestan las convenciones de puntuación en la oralidad y en la escritura; en el chat hay varias características que podrían seguirse observando y que podrían constituir una nueva 
forma de escritura a través de las herramientas de comunicación mediadas por computador. Es posible que con las interrupciones y las convenciones de puntuación en los chat y los foros puedan hacerse visibles los conflictos en las relaciones.

\section{Agradecimientos}

A la Universidad de Medellín por haber financiado la investigación "Interacciones comunicativas en un Entorno Virtual de Aprendizaje"

Al Cinde por haber permitido la participación de la maestranda coinvestigadora Claudia Vásquez Lopera.

A los estudiantes de la asignatura electiva TIC 2009-1.

A todos los demás que contribuyeron en esta investigación.

\section{Referencias bibliográficas}

Austin, John L. (1962) How to do things with words.Traducido y publicado en español por Oxford University Press. Barcelona: Ediciones Paidós.

Galeano Marín, María Eumelia (2004). Estrategias de investigación social cualitativa. El giro en la mirada. Medellín: La Carretera Editores.
MAYANS I PLANELLS, Joan (2002). Género chat o cómo la etnografía puso un pie en el ciberespacio. España: Editorial Gedisa, España.

Medina Rivilla, Antonio; Salvador Mata, Francisco (2002). Didáctica General. Madrid, España, Editorial Prentice Hall.

Restrepo Gómez, Bernando; Román Maldonado; Carlos Eduardo; Londoño Giraldo, Eliana (2009). Situación actual de la investigación y la práctica discursiva sobre la evaluación de aprendizajes en e-learning en la Educación Superior. Medellín: Católica del Norte Fundación Universitaria.

Sánchez Upegui, Alexánder Arbey; Puerta Gil, Carlos Augusto; Sánchez Ceballos, Lina María (2010). Manual de comunicación en ambientes educativos virtuales. Colombia, Fundación Universitaria Católica del Norte.

Vásquez Lopera, Claudia Patricia; Arango Vásquez, Sandra Isabel (2010). Capítulo 7: Interacciones comunicativas en un Entorno Virtual de Aprendizaje (EVA). En: Pensar la comunicación - Reflexiones y resultados de investigación, Tomo II. p. 129-146, Medellín: Sello Editorial Universidad de Medellín.

Watzlawick, Paul; Beavin, Janet H, Jackson, Don D. (1971). Teoría de la comunicación humana. Interacciones, patologías y paradojas. Argentina: Editorial Tiempo Contemporáneo. 